Z Epileptol 2012 $\cdot 25: 66-66$

DOI 10.1007/s10309-011-0231-5

Online publiziert: 25. Dezember 2011

(c) Springer-Verlag 2011

\title{
K. Wagner
}

Epilepsiezentrum, Universitätsklinikum Freiburg, Freiburg

\section{Neuropsychologie der Epilepsien}

\author{
Buchbesprechung \\ Mayer H (2011) Neuropsychologie der \\ Epilepsien. Aus der Reihe „Fortschritte der \\ Neuropsychologie", Bd. 9. Hogrefe, Göttingen. \\ Einzelpreis EUR 22,95, im Reihenabonnement \\ EUR 15,95
}

Das Übersichtsbuch zu neuropsychologischen Aspekten bei Patienten mit Epilepsien mit dem Titel Neuropsychologie der Epilepsien von Dr. Hans Mayer gibt nicht nur eine umfassende Einführung in die Erkrankung und ihre Behandlungsmöglichkeiten, sondern beschreibt auch alle relevanten Aspekte der neuropsychologischen Befunderhebung bei diesen Patienten. Das Buch beginnt mit einer umfangreichen Einführung in die Erkrankung (Epidemiologie, Ätiologie, Diagnostik, Prognose und Verlauf), einschließlich der Beschreibung verschiedener Anfallstypen und ihrer semiologischen Zeichen, der Klassifikation von Epilepsien, ihrer Differenzialdiagnostik, ihrer konservativen Behandlung sowie verschiedener nichtmedikamentöser Behandlungsoptionen. Neben den im Rahmen der Erkrankung auftretenden kognitiven Störungen befasst es sich auch mit möglichen sozialen sowie emotionalen Störungen und beschreibt psychologische Therapieansätze zur Verbesserung der Anfallskontrolle (z. B. Biofeedback). Das Buch gibt einen Überblick über die bei den wichtigsten Epilepsietypen beobachteten kognitiven Beeinträchtigungen und die dazu in der Routine- oder prächirurgischen Diagnostik häufig eingesetzten Testverfahren. Hierbei wird auch auf die Besonderheiten im Kinder- und Jugendalter eingegangen. Die Testverfahren und die von ihnen gemessenen Funktionsbereiche sind zur praktischen Nutzung ebenfalls tabellarisch auf einer herausnehmbaren Karte abgedruckt.

Besonders hilfreich ist, dass auf alle wichtigen im klinischen neuropsychologischen Alltag zu beachtenden Gesichtspunkte bei der Untersuchung von Epilepsiepatienten eingegangen wird: Hierzu gehören der Einfluss von klinischer und subklinischer epileptischer Aktivität, von strukturellen Läsionen, von psychiatrischen Begleiterkrankungen sowie von kognitiven und psychiatrischen Nebenwirkungen der gängigsten antikonvulsiven Medikamente. Den Abschluss des Buches bilden zwei Fallbeschreibungen, die nicht nur die kognitiven Profile der Patienten, sondern auch alle relevanten neurologischen und psychiatrischen Befunde darstellen. Somit gibt das anwenderfreundliche Buch als Erstes eine umfangreiche Übersicht für jeden mit Epilepsiepatienten arbeitenden Neuropsychologen und ist mit EUR 22,95 erschwinglich.

Kathrin Wagner, Freiburg

\section{Korrespondenzadresse}

Dr. K. Wagner

Epilepsiezentrum, Universitätsklinikum Freiburg Breisacher Str. 64, 79106 Freiburg

Kathrin.Wagner@uniklinik-freiburg.de

Interessenkonflikt. Die korrespondierende Autorin gibt an, dass kein Interessenkonflikt besteht. 